\section{Diabetes auto-referido em idosos: prevalência, fatores associados e práticas de controle}

\author{
Self-reported diabetes in the elderly: prevalence, \\ associated factors, and control practices
}

\begin{abstract}
The aim of the study was to assess the prevalence of self-reported diabetes in the elderly, identifying associated factors, knowledge, and practices related to treatment options. This was a crosssectional population-based study with stratified clustered two-stage sampling in six municipalities in the State of São Paulo, Brazil. Among the 1,949 elderly, 15.4\% presented self-reported diabetes. Body mass index and exercising were statistically associated with diabetes. There was a significant difference between diabetics and non-diabetics in terms of self-rated health, hospitalization, self-reported illness in the previous two weeks, and report of the following diseases: hypertension, anemia, chronic kidney disease, and heart disease. In terms of per capita family income, there was no difference in regular medical visits, participation in discussion groups, and control practices. The findings show the need for behavior changes to prevent and control diabetes and its complications. Educational interventions are needed to expand the coverage of diabetes care.
\end{abstract}

Diabetes Mellitus; Health of the Aged; Chronic Diseases
Priscila Maria Stolses Bergamo Francisco 1 Ana Paula Belon 1

Marilisa Berti de Azevedo Barros 1

Luana Carandina 2

Maria Cecília Goi Porto Alves 3

Moises Goldbaum 4

Chester Luiz Galvão Cesar ${ }^{5}$

\section{Introdução}

Entre as doenças crônicas não transmissíveis, o diabetes mellitus se destaca como importante causa de morbidade e mortalidade, especialmente entre os idosos. $\mathrm{O}$ acelerado ritmo do processo de envelhecimento da população, a maior tendência ao sedentarismo e a inadequados hábitos alimentares, além de outras mudanças sóciocomportamentais, contribuem para os crescentes níveis de incidência e prevalência do diabetes, bem como de mortalidade pela doença 1,2 .

Estimativas apontam que, enquanto em 2000 havia 171 milhões de pessoas com diabetes no mundo, em 2030 esse valor atingirá 366 milhões. Neste cenário, o Brasil terá cerca de 11,3 milhões de diabéticos ${ }^{3}$. De acordo com a Pesquisa Nacional por Amostra de Domicílios (PNAD) de 1998, a prevalência de diabetes auto-referido pela população idosa brasileira foi de 10,3\% 4. Dados da PNAD 2003 apontam na população brasileira prevalência de $12 \%$ nos homens e $16 \%$ nas mulheres, na faixa de 70 a 79 anos de idade 5 .

O diabetes, embora com menor prevalência se comparado a outras morbidades, é uma doença altamente limitante, podendo causar cegueira, amputações, nefropatias, complicações cardiovasculares e encefálicas, entre outras, que acarretam prejuízos à capacidade funcional, autonomia e qualidade de vida do indivíduo. Também é uma das principais causas de mortes prematuras, em virtude do aumento do risco para o 
desenvolvimento de doenças cardiovasculares, as quais contribuem para $50 \%$ a $80 \%$ das mortes dos diabéticos 6,7. Esses dados ilustram o impacto do alto custo social e financeiro do diabetes ao sistema de saúde, à família e à pessoa portadora da doença 1,2,6,8.

Em face desse quadro, em que o diabetes assume crescente importância na saúde pública no Brasil, delineiam-se políticas, programas e campanhas específicas que propõem medidas terapêuticas e de promoção de hábitos saudáveis para a prevenção e o controle do diabetes e de suas complicações ${ }^{8}$.

O objetivo deste estudo foi avaliar a prevalência de diabetes auto-referido em idosos residentes em diferentes áreas do Estado de São Paulo no período de 2001 a 2002, segundo características demográficas, sócio-econômicas e de estilo de vida. Objetivou-se, também, analisar a condição de saúde e a presença de co-morbidades dos idosos diabéticos, seus conhecimentos e práticas quanto às opções de tratamento e controle da doença.

\section{Métodos}

Estudo transversal de base populacional realizado com dados referentes à população de 60 anos ou mais, não institucionalizada, residente em área urbana nos municípios de Campinas e Botucatu, na região sudoeste da Grande São Paulo (Itapecerica da Serra, Embu e Taboão da Serra) e no distrito do Butantã (Município de São Paulo), no período de 2001 e 2002. Os dados foram obtidos do estudo multicêntrico Inquérito de Saúde de Base Populacional em Municípios do Estado de São Paulo (ISA-SP) 9. A escolha das áreas de estudo considerou projetos docente-assistenciais das instituições acadêmicas envolvidas na realização do inquérito.

Foi planejada amostra de 200 idosos de cada sexo em cada área. Esse tamanho de amostra foi obtido considerando-se a estimação de proporções da ordem de 0,50 , com erro de amostragem de $10 \%$, nível de $95 \%$ de confiança e efeito de delineamento de 210.

Utilizou-se amostragem estratificada de conglomerados em dois estágios. No primeiro, 30 setores censitários de cada área foram sorteados com probabilidade proporcional ao tamanho, expresso pelo número de domicílios existentes no setor, de acordo com a Contagem Populacional de 1996 do Instituto Brasileiro de Geografia e Estatística (IBGE). No segundo, uma amostra sistemática simples de domicílios foi sorteada em cada setor censitário com base na listagem dos domicílios previamente arrolados 10 .
As informações foram obtidas por meio de questionário composto por questões, na sua maioria, fechadas, organizadas em blocos temáticos, a saber: condições de vida, estilo de vida, percepção e qualidade de saúde, morbidade auto-referida, uso de serviços de saúde e consumo de medicamentos.

A variável dependente deste estudo foi o diabetes mellitus auto-referido (sim ou não) e as variáveis independentes analisadas foram características demográficas, sócio-econômicas e de estilo de vida. Foi também estudada a distribuição de indicadores de condição de saúde, segundo a presença do diabetes, bem como o uso de serviços de saúde, conhecimentos e práticas quanto ao tratamento da doença, em relação à renda familiar per capita dos idosos diabéticos.

- Variáveis demográficas e sócio-econômicas: sexo, idade, situação conjugal, escolaridade (em anos de estudo), atividade de trabalho e renda familiar per capita (em salários mínimos).

- Variáveis de estilo de vida: tabagismo, prática de atividade física no lazer (obtida por meio da pergunta "Pratica regularmente, pelo menos uma vez por semana, algum esporte ou exercício físico?") e índice de massa corporal (IMC) com os seguintes pontos de corte recomendados para o idoso 11: baixo peso $\left(<22 \mathrm{~kg} / \mathrm{m}^{2}\right)$, eutrofia $(22 \leq$ IMC $\left.\leq 27 \mathrm{~kg} / \mathrm{m}^{2}\right)$ e sobrepeso ( $\left.>27 \mathrm{~kg} / \mathrm{m}^{2}\right) ;$ o IMC foi calculado com dados de peso e altura autoreferidos.

- Indicadores de condição de saúde: percepção da própria saúde, internação hospitalar nos últimos 12 meses, morbidade auto-referida nos 15 dias que antecederam a pesquisa (sim ou não) e relato (informação obtida por meio de checklist) das seguintes doenças: hipertensão, anemia, doença renal crônica, depressão/ansiedade/problemas emocionais e doença do coração.

- Uso de serviços de saúde, conhecimento e conduta em relação ao tratamento: somente os que referiram diabetes responderam um bloco à parte, com questões sobre quem disse que o entrevistado era diabético, com que idade soube ser diabético, o que fazia para controlar a doença, se visitava o médico/serviço de saúde periodicamente por causa do diabetes, se havia participado de grupos de discussão sobre o controle da enfermidade e o que sabia a respeito do que deveria ser feito para controlar o diabetes. Os motivos pelos quais os idosos diabéticos não visitam o médico/serviço de saúde foram descritos com base na seguinte pergunta: "Por que o(a) sr(a) não visita o médico/serviço de saúde regularmente por causa do diabetes?". O status vacinal contra gripe entre os idosos diabéticos também foi avaliado.

As análises foram realizadas utilizando-se procedimentos para análise de inquéritos popu- 
lacionais com amostras complexas do programa Stata 8.0 (Stata Corp., College Station, Estados Unidos), os quais incorporam as ponderações necessárias e consideram a existência da homogeneidade intraconglomerado, produzindo estimativas não viciadas do erro-padrão. A associação entre as diversas variáveis e a presença de diabetes foi verificada na análise bivariada pelo teste $\chi^{2}$, com nível de $5 \%$ de significância. Foram utilizadas razões de prevalência e intervalos de 95\% de confiança (IC95\%), calculados por meio de regressão de Poisson ${ }^{12}$. Modelos de regressão múltipla de Poisson foram desenvolvidos para ajustes por idade e sexo.

O projeto deste inquérito foi aprovado pelo Comitê de Ética em Pesquisa da Faculdade de Ciências Médicas da Universidade Estadual de Campinas, sob o parecer no. $369 / 2000$.

\section{Resultados}

Nos domicílios visitados em que foi possível obter dados sobre os moradores, foram localizados 2.151 idosos com idade igual ou superior a 60 anos. Entre os idosos com resposta válida para diabetes mellitus auto-referido localizados nos domicílios sorteados, houve 9,4\% de perdas, sendo $9,1 \%$ de recusas e $0,3 \%$ por outros motivos.

Dos 1.949 idosos entrevistados, 1.025 (57,3\%) eram mulheres; a média de idade foi de 69,7 anos (IC95\%:69,0-70,4). A prevalência estimada de diabetes mellitus auto-referido foi de 14,9\% (IC95\%: 12,2-18,2) e 15,8\% (IC95\%: 12,8-19,2), respectivamente nos homens e nas mulheres (Tabela 1); 98,5\% dos idosos diabéticos referiram que o diagnóstico da doença foi feito por médico.

Não houve associação estatística significativa entre as variáveis demográficas e sócio-econômicas e diabetes auto-referido (Tabela 1). Dentre os indicadores de estilo de vida, o IMC esteve associado ao diabetes, e a prática de atividade física

Prevalência e razão de prevalência (RP) de diabetes auto-referido, segundo variáveis demográficas e sócio-econômicas em pessoas com 60 anos ou mais. Inquérito de Saúde de Base Populacional em Municípios do Estado de São Paulo (ISA-SP), São Paulo, Brasil, 2001-2002.

\begin{tabular}{|c|c|c|c|c|}
\hline Variáveis/Categorias & $n$ * & Prevalência (\%) & Valor de $p * \star$ & RP (IC95\%) \\
\hline \multicolumn{5}{|l|}{ Sexo } \\
\hline Masculino & 924 & 14,9 & & 1 \\
\hline Feminino & 1.025 & 15,8 & 0,71 & $1,06(0,78-1,42)$ \\
\hline \multicolumn{5}{|l|}{ Faixa etária (anos) } \\
\hline $60-69$ & 1.086 & 15,7 & & 1 \\
\hline $70-79$ & 642 & 15,1 & & $0,96(0,65-1,42)$ \\
\hline 80 ou mais & 221 & 14,7 & 0,95 & $0,94(0,59-1,48)$ \\
\hline \multicolumn{5}{|l|}{ Situação conjugal } \\
\hline Casado/União estável & 1.153 & 17,4 & & 1 \\
\hline Solteiro & 227 & 12,6 & & $0,72(0,38-1,39)$ \\
\hline Viúvo & 565 & 12,7 & 0,21 & $0,73(0,51-1,04)$ \\
\hline \multicolumn{5}{|l|}{ Escolaridade (anos) } \\
\hline Até 4 & 1.403 & 15,7 & & 1 \\
\hline 5 ou mais & 545 & 14,9 & 0,79 & $0,95(0,66-1,37)$ \\
\hline \multicolumn{5}{|c|}{ Renda per capita (salários mínimos ${ }^{\star \star \star}$ ) } \\
\hline$\leq 2,5$ & 1.180 & 14,8 & & 1 \\
\hline$>2,5$ & 769 & 16,3 & 0,49 & $1,11(0,83-1,48)$ \\
\hline \multicolumn{5}{|l|}{ Atividade de trabalho } \\
\hline Sim & 498 & 15,9 & & 1 \\
\hline Não & 1.451 & 15,3 & 0,80 & $0,96(0,69-1,34)$ \\
\hline
\end{tabular}

\footnotetext{
* Número de indivíduos na amostra não ponderada;

** Valor de $p$ do teste $\chi^{2}$;

*** Salário mínimo vigente à época da pesquisa: março a abril/2001 = R\$151,00; maio/2001 a março/2002 = R\$ 180,00 e abril a setembro/2002 $=\mathrm{R} \$ 200,00$.
} 
em contexto de lazer apresentou-se no limite da significância estatística (Tabela 2).

Na comparação entre idosos diabéticos e não diabéticos quanto às condições de saúde avaliadas neste estudo, foi possível observar perfis diferenciados. No que se refere à percepção da própria saúde, houve diferença estatisticamente significativa entre os grupos. Entre os idosos diabéticos, $23,7 \%$ consideraram sua saúde ruim ou muito ruim, enquanto o percentual entre os não diabéticos foi de apenas $11,2 \%$. Entre os que referiram internação hospitalar nos 12 meses que antecederam a pesquisa, a proporção observada foi de $19,6 \%$ nos diabéticos e 9,7\% nos não diabéticos. Também em relação à morbidade nos últimos 15 dias, a prevalência foi maior entre os diabéticos. Já para as condições crônicas autoreferidas, a proporção de doença renal crônica foi cerca de três vezes maior entre os idosos diabéticos, e apenas o relato de depressão/ansiedade/problemas emocionais não esteve associado à doença (Tabela 3).

Em relação ao uso de serviços de saúde, aos conhecimentos e às práticas de controle da doença, verificou-se que $75,2 \%$ dos idosos diabéticos visitam periodicamente o médico/serviço de saúde. Dentre os idosos que não procuram regularmente, cerca de $55 \%$ não o faz por achar desnecessário. Dos idosos diabéticos avaliados neste estudo, $56,5 \%$ e $19 \%$ referiram o uso de medicamento oral e insulina de rotina, respectivamente. Quanto à vacinação contra gripe, 75,6\% dos idosos diabéticos referiram imunização nos 12 meses que antecederam a pesquisa. Não hou- ve associação estatística entre a renda familiar per capita e as variáveis analisadas sobre o uso de serviços de saúde, conhecimento e práticas de controle da doença (Tabela 4).

\section{Discussão}

Neste estudo, a prevalência de diabetes mellitus auto-referido em idosos com 60 anos de idade ou mais foi estimada em 15,4\% (IC95\%: 13,3-17,6). Em pesquisa realizada pelo Instituto Nacional de Câncer (INCA) 13, realizada em 15 capitais brasileiras e no Distrito Federal, foi encontrado resultado similar para esse mesmo segmento populacional, com prevalência auto-referida de 14\% (IC95\%: 11,6-25,2). No Projeto Bambuí - Estudo de Coorte de Base Populacional da Saúde dos Idosos 14, em que foram aplicados testes clínicos para o diagnóstico da doença, a prevalência de diabetes foi de $14,6 \%$. No Projeto SABE - Saúde, Bem-Estar e Envelhecimento, a prevalência do diabetes auto-referido pela população idosa residente no Município de São Paulo foi estimada em $17,9 \% 15$.

Constitui uma das limitações da presente investigação o fato de que a informação utilizada para estimar a prevalência de diabetes é auto-referida, sem testes clínicos para comprovar o diagnóstico da doença. Diversos estudos apontam que a acurácia da informação auto-referida de morbidade varia conforme a doença, a sua gravidade, a presença de co-morbidades e características sócio-econômicas e demográficas 5,16,17,18.

Tabela 2

Prevalência e razão de prevalência (RP) de diabetes auto-referido, segundo estilo de vida em pessoas com 60 anos ou mais. Inquérito de Saúde de Base Populacional em Municípios do Estado de São Paulo (ISA-SP), São Paulo, Brasil, $2001-2002$.

\begin{tabular}{|c|c|c|c|c|}
\hline Variáveis/Categorias & $n$ * & Prevalência (\%) & Valor de $p * *$ & RP (IC95\%) \\
\hline \multicolumn{5}{|l|}{ Tabagismo } \\
\hline Não fumante & 1.038 & 15,9 & & 1 \\
\hline Fumante & 289 & 10,9 & & $0,68(0,42-1,11)$ \\
\hline Ex-fumante & 618 & 16,5 & 0,24 & $1,04(0,78-1,38)$ \\
\hline \multicolumn{5}{|c|}{ Índice de massa corporal (kg/m²) } \\
\hline$<22$ & 293 & 6,6 & & 1 \\
\hline $22-27$ & 787 & 14,9 & & $2,26(1,15-4,45)$ \\
\hline$>27$ & 575 & 21,3 & $<0,01$ & $3,24(1,61-6,51)$ \\
\hline \multicolumn{5}{|c|}{ Prática de atividade física no lazer } \\
\hline Sim & 607 & 11,7 & & 1 \\
\hline Não & 1.337 & 16,9 & 0,06 & $1,44(0,98-2,11)$ \\
\hline
\end{tabular}

* Número de indivíduos na amostra não ponderada;

** Valor de $p$ do teste $\chi^{2}$. 
Condição de saúde segundo presença de diabetes auto-referido em pessoas com 60 anos ou mais. Inquérito de Saúde de Base Populacional em Municípios do Estado de São Paulo (ISA-SP), São Paulo, Brasil, 2001-2002.

\begin{tabular}{|c|c|c|c|c|c|c|c|}
\hline \multirow[t]{2}{*}{ Variáveis/Categorias } & \multicolumn{2}{|c|}{ Diabéticos } & \multicolumn{2}{|c|}{ Não-diabéticos } & \multicolumn{2}{|c|}{ Total } & \multirow{2}{*}{$\begin{array}{l}\text { Valor } \\
\text { de } p \text { ** }\end{array}$} \\
\hline & $n$ * & $\%$ & $n$ * & $\%$ & $n$ * & $\%$ & \\
\hline \multicolumn{8}{|l|}{ Percepção da própria saúde } \\
\hline Excelente/Muito boa & 47 & 16,6 & 414 & 28,8 & 461 & 26,9 & \\
\hline Boa & 170 & 59,7 & 995 & 60,0 & 1.165 & 60,0 & \\
\hline Ruim/Muito ruim & 69 & 23,7 & 204 & 11,2 & 273 & 13,1 & $<0,01$ \\
\hline \multicolumn{8}{|l|}{ Internação hospitalar nos últimos 12 meses } \\
\hline Nenhuma & 233 & 80,4 & 1.485 & 90,3 & 1.718 & 88,8 & \\
\hline 1 ou mais & 59 & 19,6 & 171 & 9,7 & 230 & 11,2 & $<0,01$ \\
\hline \multicolumn{8}{|l|}{ Morbidade nos últimos 15 dias } \\
\hline Não & 189 & 67,8 & 1.216 & 76,6 & 1.405 & 75,3 & \\
\hline $\operatorname{Sim}$ & 103 & 32,2 & 441 & 23,4 & 544 & 24,7 & 0,02 \\
\hline \multicolumn{8}{|l|}{ Relato das seguintes condições/doenças crônicas } \\
\hline Hipertensão & 190 & 67,9 & 745 & 47,9 & 935 & 51,0 & $<0,01$ \\
\hline Anemia & 20 & 7,2 & 72 & 3,6 & 92 & 4,2 & 0,04 \\
\hline Doença renal crônica & 34 & 10,2 & 85 & 3,4 & 119 & 4,5 & $<0,01$ \\
\hline Depressão, ansiedade e problemas emocionais & 79 & 28,8 & 393 & 23,8 & 472 & 24,5 & 0,23 \\
\hline Doença do coração & 61 & 20,8 & 228 & 14,9 & 289 & 15,8 & 0,04 \\
\hline
\end{tabular}

* Número de indivíduos na amostra não ponderada;

** Valor de $p$ do teste $\chi^{2}$.

Okura et al. 16, em estudo realizado no Estado de Minnesota, Estados Unidos, entre pessoas de 45 anos ou mais, verificaram, para o diabetes, $99,7 \%$ de especificidade e $66 \%$ de sensibilidade. LimaCosta et al. 17, investigando a validade da informação auto-referida entre os idosos que participaram do Projeto Bambuí, registraram sensibilidade de $57,1 \%$ e alta especificidade (96\%) para a doença. Portanto, no presente estudo, cuja informação é auto-referida, a prevalência do diabetes sofre subestimação.

Em relação às características sócio-demográficas, verificou-se não haver diferença estatisticamente significativa entre os sexos, conforme apontado por Passos et al. 14. Observou-se ligeira diminuição não significativa da prevalência de diabetes com o aumento da idade. Essa relação inversa não significativa foi encontrada também pelo Projeto SABE 15. Uma possível explicação para o não-aumento da prevalência do diabetes com o progredir da idade repousaria no viés de sobrevivência, uma vez que aqueles mais vulneráveis às complicações geradas pela doença teriam maior probabilidade de morrer prematuramente 19 .

Já as variáveis escolaridade, renda familiar per capita e atividade de trabalho não apresentaram associação estatística significativa com o diabe- tes, mesmo quando ajustadas por idade e sexo (dados não apresentados), indicando que, entre os idosos, a prevalência da doença não é influenciada por fatores sócio-econômicos. Entretanto, associação inversa entre diabetes e escolaridade foi observada na população brasileira de 18 anos ou mais com dados da PNAD 5 e na população de 20 anos ou mais do Município de Campinas 20. Para a população idosa, o Projeto Bambuí verificou associação estatisticamente significativa entre diabetes e baixos níveis educacionais e de renda ${ }^{14}$. A não-existência de diferenças sócioeconômicas na prevalência de diabetes no presente estudo poderia ser resultante do viés de sobrevivência, visto que pessoas diabéticas com piores condições de vida teriam maior risco de morrer precocemente 19 .

Um achado importante deste trabalho é que, apesar das incapacidades e limitações que a doença pode causar, $16,6 \%$ dos idosos diabéticos consideraram sua saúde como excelente/muito boa, e 59,7\%, como boa. Uma possível explicação para essa situação é que, nesta amostra, a maioria dos idosos diabéticos $(76,2 \%)$ não relatou complicações decorrentes da doença (como problemas de visão, renais, circulatórios e outros), as quais poderiam comprometer mais a avaliação do estado de saúde. Sem dúvida, o maior contro- 
Uso de serviços de saúde, conhecimento e práticas quanto às opções do tratamento do diabetes, segundo renda familiar per capita de pessoas com 60 anos ou mais. Inquérito de Saúde de Base Populacional em Municípios do Estado de São Paulo (ISA-SP), São Paulo, Brasil, $2001-2002$.

\begin{tabular}{|c|c|c|c|c|c|c|c|}
\hline \multirow[t]{3}{*}{ Total } & \multicolumn{6}{|c|}{ Renda familiar per capita (salários mínimos) } & \multirow[t]{3}{*}{ Valor de $p$} \\
\hline & \multirow[b]{2}{*}{$n$ * } & \multicolumn{3}{|c|}{$\leq 2,5$} & \multicolumn{2}{|c|}{$>2,5$} & \\
\hline & & $\%$ & $n$ * & $\%$ & $n$ * & $\%$ & \\
\hline \multicolumn{8}{|l|}{ Com que idade o médico disse que era } \\
\hline \multicolumn{8}{|l|}{ diabético (anos) } \\
\hline $0-19$ & 67 & 19,9 & 43 & 20,2 & 24 & 19,5 & 0,30 \\
\hline $20-59$ & 104 & 37,6 & 57 & 32,5 & 47 & 44,0 & \\
\hline 60 ou mais & 108 & 42,5 & 64 & 47,3 & 44 & 36,5 & \\
\hline Total & 279 & & 164 & & 115 & & \\
\hline \multicolumn{8}{|l|}{ Visita periodicamente médico/serviço de saúde } \\
\hline \multicolumn{8}{|l|}{ por causa do diabetes } \\
\hline Não & 69 & 24,8 & 46 & 26,5 & 23 & 22,6 & 0,59 \\
\hline Sim & 220 & 75,2 & 124 & 73,5 & 96 & 77,4 & \\
\hline Total & 289 & & 170 & & 119 & & \\
\hline \multicolumn{8}{|l|}{ Participa de grupos de discussão sobre diabetes } \\
\hline Não & 245 & 86,4 & 142 & 85,7 & 103 & 87,3 & 0,76 \\
\hline $\operatorname{Sim}$ & 41 & 13,6 & 27 & 14,3 & 14 & 12,7 & \\
\hline Total & 286 & & 169 & & 117 & & \\
\hline \multicolumn{8}{|l|}{ O que acha que deve ser feito para controlar o diabetes ** } \\
\hline Dieta alimentar e/ou regime para perder/manter peso & 203 & 68,6 & 116 & 65,4 & 87 & 72,8 & 0,29 \\
\hline Tomar medicamento oral de rotina & 129 & 44,9 & 73 & 45,9 & 56 & 43,6 & 0,74 \\
\hline Tomar insulina de rotina & 34 & 12,9 & 21 & 11,6 & 13 & 14,5 & 0,56 \\
\hline \multicolumn{8}{|l|}{ O que faz para controlar o diabetes ** } \\
\hline Dieta alimentar ou regime para perder/manter peso & 169 & 62,8 & 93 & 58,1 & 76 & 68,8 & 0,23 \\
\hline Toma medicamento oral de rotina & 168 & 56,5 & 99 & 57,4 & 69 & 55,3 & 0,77 \\
\hline Toma insulina de rotina & 54 & 19,0 & 31 & 19,7 & 23 & 18,1 & 0,81 \\
\hline \multicolumn{8}{|l|}{ Tem alguma complicação por causa do diabetes } \\
\hline Não & 221 & 76,2 & 129 & 78,3 & 92 & 73,5 & 0,50 \\
\hline Sim & 64 & 23,8 & 37 & 21,7 & 27 & 26,5 & \\
\hline Total & 285 & & 166 & & 119 & & \\
\hline \multicolumn{8}{|l|}{ Tomou vacina contra gripe nos últimos 12 meses } \\
\hline Não & 87 & 24,4 & 45 & 20,8 & 42 & 28,9 & 0,12 \\
\hline Sim & 197 & 75,6 & 121 & 79,2 & 76 & 71,1 & \\
\hline Total & 284 & & 166 & & 118 & & \\
\hline
\end{tabular}

* Número absoluto de indivíduos na amostra não ponderada;

** Questões que permitiam mais de uma resposta.

le da doença, aliado ao avanço de medidas terapêuticas, contribui para o não-agravamento das condições de saúde dos idosos diabéticos. Além disso, cerca de $16 \%$ dos idosos diabéticos referiram exercer alguma atividade de trabalho, remunerada ou não. No entanto, deve-se considerar também a importância, para a renda familiar, da contribuição do idoso, oriunda seja de aposentadoria ou pensões, seja de sua participação no mercado de trabalho ${ }^{21}$, já que tal contribuição poderia justificar a permanência do idoso no exercício de alguma atividade de trabalho.
É importante salientar que este estudo, por ser de desenho transversal, não permite em geral identificar os vínculos causais entre os eventos. Portanto, não é permitido distinguir se os fatores associados ao diabetes seriam causas ou conseqüências da própria doença.

A obesidade foi mensurada a partir do IMC com peso e altura auto-referidos. O ponto de corte utilizado é o IMC $>27 \mathrm{~kg} / \mathrm{m}^{2}$, recomendado para o idoso por considerar as modificações na composição corporal decorrentes do envelhecimento, tais como diminuição óssea, muscular e 
da água corporal, além de aumento e redistribuição da gordura 11. O IMC mostrou-se significativamente associado ao diabetes, o que corrobora os achados de diversos estudos que registraram a relação estatisticamente significativa entre obesidade e incidência ou prevalência de diabetes entre idosos 14,22,23. A literatura médico-científica reconhece a obesidade como um fator de risco à incidência da doença e recomenda fortemente a diminuição e o controle do peso como uma das principais estratégias de tratamento não farmacológico do diabetes 1,2. A Sociedade Brasileira de Diabetes ${ }^{1}$ aponta que a perda de $5 \%$ a $10 \%$ de peso possibilitaria reduzir os níveis glicêmicos, retardar a progressão da doença, diminuir as necessidades insulínicas e, inclusive, permitiria retirar o tratamento farmacológico.

Embora no limite da significância estatística, os dados de prática de atividade física em contexto de lazer deste estudo apontam prevalência de diabetes maior entre os sedentários, assim como observado em diferentes estudos longitudinais e transversais 1,6 .

Como o sedentarismo mostra-se fortemente associado ao desenvolvimento de doenças crônicas não transmissíveis, entre elas o diabetes, a prática de atividades físicas é apontada como uma importante estratégia para a prevenção da doença 1,2 . Neste estudo, optou-se por restringir a investigação à atividade física no lazer, desconsiderando as outras dimensões da atividade física global, as quais abrangem aquelas desempenhadas no trabalho, no deslocamento e no meio doméstico. Considera-se a atividade física praticada no lazer um indicador mais adequado por representar a adoção de estilo de vida saudável e ser alvo de políticas públicas de saúde. Contudo, cabe acrescentar que a definição de sedentário no lazer excluiu aqueles que praticaram atividade no mínimo uma vez por semana e que, por isso, não eram suficientemente ativos 24 .

Tendo em vista que a elevada e crescente prevalência da obesidade no contexto global é resultado tanto do maior consumo de alimentos com alto teor de açúcares e gorduras saturadas, como da inatividade física ${ }^{2}$, os programas e profissionais de saúde têm defendido a adoção de estilo de vida mais saudável (mudanças de hábitos alimentares, aumento da atividade física diária e abandono do tabagismo) no combate não apenas ao diabetes, mas também a outras doenças crônicas e incapacidades funcionais. A despeito das divergências entre as pesquisas no que se refere à eficácia da combinação entre dieta alimentar e prática de atividade física na redução da incidência ou no maior controle da doença 25,26, é consenso que a modificação no estilo de vida é de extrema importância, sobretudo, entre a população idosa que registra um aumento dos índices de obesidade 23 e maior incidência de comorbidades.

A maior prevalência significativa de hipertensão entre os diabéticos, verificada neste estudo, era esperada, em face das pesquisas clínicas que comprovam a relação entre tais enfermidades. Como a hipertensão está associada a um maior grau de resistência à insulina, e os medicamentos anti-hipertensivos podem agravar este quadro, o hipertenso torna-se mais suscetível a desenvolver diabetes 1,27 .

Estudos de base transversal também registraram esta relação. Souza et al. 22 estimaram que o risco de apresentar diabetes é três vezes maior nos indivíduos hipertensos. Essa estreita associação é preocupante, tendo em vista que a hipertensão nos diabéticos aumenta o risco de complicações cardiovasculares, como acidente vascular cerebral e aterosclerose 15,22. O diabetes constitui ainda um fator de risco para a doença arterial coronariana e pode contribuir para o desenvolvimento de hipertrofia ventricular esquerda com evolução para insuficiência cardíaca 28 . Neste estudo, houve associação estatística significativa entre diabetes auto-referido e doença do coração, sendo que em torno de $21 \%$ e $15 \%$ dos idosos diabéticos e não diabéticos, respectivamente, relataram a doença.

A associação significativa entre diabetes e doença renal crônica, também observada neste estudo, pode ser explicada pela maior predisposição do diabético em desenvolver alguma nefropatia. Conforme Romão Junior 29, o risco de desenvolver nefropatia é $30 \%$ maior nos diabéticos do tipo 1, e $20 \%$ maior nos diabéticos do tipo 2. A nefropatia diabética pode conduzir à insuficiência renal crônica, o que geraria a necessidade de hemodiálise ou mesmo transplante de rim, aumentando o ônus desta doença. Além disso, é sabido que a hipertensão e o diabetes são as duas principais causas de insuficiência renal crônica e que a primeira se torna um fator de risco para complicações renais entre os diabéticos 27,29.

A anemia também foi mais prevalente entre os diabéticos. A literatura aponta que a anemia é uma doença comum entre os diabéticos e pode favorecer o aparecimento de complicações 30 , principalmente naqueles portadores de doença renal crônica 28.

Considerando que as informações de todas as doenças incluídas nesta pesquisa são auto-referidas e que, portanto, dependeriam do acesso aos serviços de saúde para a realização de diagnóstico, os pacientes que freqüentam o serviço para tratar de uma enfermidade teriam maior probabilidade de ter diagnosticadas outras doenças. Desse modo, deve-se considerar que a maior 
prevalência de outras doenças entre os diabéticos poderia também decorrer desse viés.

A maior prevalência de percepção da própria saúde como ruim ou muito ruim entre os diabéticos, comparados aos não diabéticos, pode decorrer do fato de que essa doença, em alguns casos, impõe limitações ao indivíduo e, muitas vezes, pode gerar seqüelas que impossibilitam relatos favoráveis dos idosos acerca de sua condição de saúde. A descompensação do diabetes mellitus e a instabilidade de seu quadro, quando não acompanhadas por tratamento adequado, podem influir na avaliação da saúde ${ }^{8}$. Estudo utilizando o SF-36 (Medical Outcomes Study 36-Item Short-Form Health Survey) apontou, nos idosos com diabetes, pior qualidade de vida em saúde em todas as dimensões avaliadas pelo instrumento, em especial em relação à capacidade funcional e ao estado geral de saúde 31 . Vale ressaltar que a auto-avaliação da saúde se apresenta como um indicador robusto do estado de saúde e como um preditor consistente da sobrevivência dos idosos 32. Todavia, não houve associação estatística significativa entre diabetes e transtornos psicológicos, mensurados neste estudo por depressão, ansiedade e/ou problemas emocionais auto-referidos.

A maior susceptibilidade dos diabéticos ao desenvolvimento de outras doenças e/ou agravos à saúde provavelmente favorece, ainda, o maior relato de morbidade nos 15 dias que antecederam a pesquisa $(32,2 \%)$ e a alta prevalência de internação hospitalar auto-referida (19,6\%) entre os diabéticos.

Em relação à vacinação contra gripe, apesar de não haver diferença estatística significativa, a maior proporção foi observada entre os idosos diabéticos de menor renda familiar per capita $(79,2 \%)$, confirmando a abrangência que as campanhas nacionais de vacinação têm tido, especialmente entre aqueles de menor nível sócio-econômico ${ }^{33}$. A Organização Mundial da Saúde (OMS) recomenda a vacinação para todos os idosos e destaca sua importância para os portadores de doenças crônicas, como doença pulmonar, cardiovascular, renal e metabólica, diante da maior gravidade das complicações clínicas decorrentes da infecção pelo vírus da influenza 34

Neste estudo, os resultados indicaram que os serviços de saúde estão garantindo o acesso ao atendimento médico a $75,2 \%$ dos idosos diabéticos, os quais referiram visitar o médico/ serviço de saúde periodicamente, sem diferença significativa entre os de menor e maior renda familiar per capita. No entanto, deve-se considerar que houve redução do poder do teste por causa do menor tamanho da amostra nesta análise. Dentre os diabéticos que não procuraram regularmente o serviço, a dificuldade financeira ou de acesso geográfico foi referida por apenas $3,1 \%$ e $5,3 \%$ dos idosos, respectivamente. Já o principal motivo apontado foi não considerar necessário (em torno de 55\%). Sobre o conhecimento e práticas quanto às opções de tratamento, $19 \%$ e $56,5 \%$ referiram que aplicam insulina e tomam medicamento oral de rotina respectivamente, apontando a necessidade de ampliação da cobertura do cuidado aos pacientes já diagnosticados.

A oferta de intervenções educativas pelos serviços de saúde e a participação dos diabéticos em grupos de discussão, com informações sobre a doença, condutas para perda de peso corporal e adoção de estilo de vida mais saudável, é essencial, por proporcionar conhecimento e habilidades aos pacientes acerca do cuidado diário que a doença demanda.

O presente artigo mostrou que, comparativamente aos não diabéticos, os idosos diabéticos apresentam maior proporção de obesidade e sobrepeso, referindo também maior percentual de internação hospitalar nos últimos 12 meses, pior avaliação de saúde, maior prevalência de morbidade nos 15 dias anteriores à pesquisa, hipertensão arterial, anemia, doenças do coração e doença renal crônica. Tais resultados indicam que, paralelamente aos investimentos no tratamento e cuidado adequado dos pacientes, são também fundamentais os programas e as campanhas governamentais com o intuito de incentivar mudanças comportamentais que favoreçam a redução dos altos níveis de incidência e de suas complicações na população idosa. 


\section{Resumo}

O objetivo do estudo foi avaliar a prevalência de diabetes auto-referido em idosos, identificando fatores associados, conhecimento e práticas quanto às opções de tratamento. Trata-se de estudo transversal de base populacional, com amostra estratificada por conglomerados e em dois estágios, em municípios do Estado de São Paulo, Brasil. Dos 1.949 idosos, 15,4\% referiram diabetes. O índice de massa corporal e a prática de exercício físico estiveram associados à doença. Houve diferença entre diabéticos e não diabéticos quanto à auto-avaliação da saúde, internação, morbidade auto-referida nos últimos 15 dias, e relato das seguintes doenças: hipertensão, anemia, doença renal e cardiovascular. Não houve desigualdade em relação à renda familiar per capita quanto à visita ao médico/serviço de saúde, à participação em grupos de discussão e às práticas de controle da doença. O estudo sugere a importância de mudanças comportamentais, como estratégias de prevenção e controle da doença e suas complicações, bem como a necessidade de oferta de intervenções educativas com ampliação da cobertura de cuidados aos diabéticos.

Diabetes Mellitus; Saúde do Idoso; Doenças Crônicas

\section{Colaboradores}

P. M. S. B. Francisco, A. P. Belon e M. B. A. Barros realizaram a proposta do artigo, revisão de literatura, análise dos dados e redação do texto. L. Carandina, M. C. G. P. Alves, M. Goldbaum e C. L. G. Cesar contribuíram na revisão do artigo.

\section{Agradecimentos}

À Fundação de Amparo à Pesquisa do Estado de São Paulo (FAPESP; processo no. 88/14099-7) e à Secretaria Estadual de Saúde de São Paulo, pelo financiamento do trabalho de campo; à Secretaria de Vigilância em Saúde do Ministério da Saúde, pelo suporte financeiro para a análise dos dados por intermédio do Centro Colaborador em Análise de Situação de Saúde da Faculdade de Ciências Médicas da Universidade Estadual de Campinas; à Coordenação de Aperfeiçoamento de Pessoal de Nível Superior (CAPES), pela bolsa de doutorado recebida por A. P. Belon; ao Conselho Nacional de Desenvolvimento Científico e Tecnológico (CNPq), pela bolsa de produtividade de M. B. A. Barros, M. Goldbaum e C. L. G. Cesar.

\section{Referências}

1. Sociedade Brasileira de Diabetes. Atualização brasileira sobre diabetes. Rio de Janeiro: Diagraphic; 2005.

2. Organização Pan-Americana da Saúde. Doenças crônico-degenerativas e obesidade: estratégia mundial sobre alimentação saudável, atividade física e saúde. Brasília: Organização Pan-Americana da Saúde; 2003.

3. Wild S, Roglic G, Green A, Sicree R, King H. Global prevalence of diabetes: estimates for the year 2000 and projections for 2030. Diabetes Care 2004; 27:1047-53.

4. Lima-Costa MF, Barreto SM, Giatti L. Condições de saúde, capacidade funcional, uso de serviços de saúde e gastos com medicamentos da população idosa brasileira: um estudo descritivo baseado na Pesquisa Nacional por Amostra de Domicílios. Cad Saúde Pública 2003; 19:735-43.
5. Barros MBA, Cesar CLG, Carandina L, Torre GD. Desigualdades sociais na prevalência de doenças crônicas no Brasil, PNAD-2003. Ciênc Saúde Coletiva 2006; 11:911-26.

6. Organização Mundial da Saúde/Federação Internacional de Diabetes. Ação já contra o diabetes: uma iniciativa da Organização Mundial da Saúde e da Federação Internacional de Diabetes. Genebra: Organização Mundial da Saúde/Federação Internacional de Diabetes; 2004.

7. Schaan BD, Harzheim E, Gus I. Perfil de risco cardíaco no diabetes mellitus e na glicemia de jejum alterada. Rev Saúde Pública 2004; 38:529-36.

8. Toscano CM. National screening campaigns for chronic non-communicable diseases: diabetes and hypertension. Ciênc Saúde Coletiva 2004; 9:885-95. 
9. Cesar CLG. Metodologia. In: Cesar CLG, Carandina L, Alves MCGP, Barros MBA, Goldbaum M, organizadores. Saúde e condição de vida em São Paulo: inquérito multicêntrico de saúde no Estado de São Paulo. São Paulo: Faculdade de Saúde Pública, Universidade de São Paulo; 2005. p. 37-46.

10. Alves MCGP. Plano de amostragem. In: Cesar CLG, Carandina L, Alves MCGP, Barros MBA, Goldbaum M. Saúde e condição de vida em São Paulo: inquérito multicêntrico de saúde no Estado de São Paulo. São Paulo: Faculdade de Saúde Pública, Universidade de São Paulo; 2005. p. 47-62.

11. Cervi A, Franceschni SCC, Priore SE. Análise crítica do uso do índice de massa corporal para idosos. Rev Nutr 2005; 18:765-75.

12. Francisco PMSB, Donalisio MR, Barros MBA, Cesar CLG, Carandina L, Goldbaum M. Medidas de associação em estudo transversal com delineamento complexo: razão de chances e razão de prevalência. Rev Bras Epidemiol 2008; 11:347-55.

13. Instituto Nacional de Câncer. Inquérito domiciliar sobre comportamentos de risco e morbidade referida de doenças e agravos não transmissíveis. Brasil, 15 capitais e Distrito Federal 2002-2003. Rio de Janeiro: Instituto Nacional de Câncer; 2003.

14. Passos VMA, Barreto SM, Diniz LM, Lima-Costa MF Type 2 diabetes: prevalence and associated factors in a Brazilian community - the Bambuí health and aging study. São Paulo Med J 2005; 123:66-71.

15. Lebrão ML. Condições de saúde. In: Lebrão ML, Laurenti R, organizadores. SABE - Saúde, Bem-estar e Envelhecimento. O Projeto Sabe no Município de São Paulo: uma abordagem inicial. Brasília: Organização Pan-Americana da Saúde; 2003. p. 73-91.

16. Okura Y, Urban LH, Mahoney DW, Jacobsen SJ, Rodeheffer RJ. Agreement between self-reported questionnaires and medical record data was substantial for diabetes, hypertension, myocardial infarction and stroke but not for heart failure. J Clin Epidemiol 2004; 57:1096-103.

17. Lima-Costa MF, Peixoto SV, Firmo JOA, Uchoa E. Validade do diabetes auto-referido e seus determinantes: evidências do projeto Bambuí. Rev Saúde Pública 2007; 41:947-53.

18. Cricelli C, Mazzaglia G, Samani F, Marchi M, Sabatini A, Nardi R, et al. Prevalence estimates for chronic diseases in Italy: exploring the differences between self-report and primary care databases. J Public Health Med 2003; 25:254-7.

19. Lima-Costa MF, Guerra HL, Firmo JOA, Uchôa E. Projeto Bambuí: um estudo epidemiológico de características sociodemográficas, suporte social e indicadores de condição de saúde dos idosos em comparação aos adultos jovens. Inf Epidemiol SUS 2002; 11:91-105.

20. Carandina L. Morbidade referida. In: Barros MBA, Cesar CLG, Carandina L, Goldbaum M, organizadores. As dimensões da saúde: inquérito populacional em Campinas. São Paulo: Aderaldo \& Rothschild; 2008. p. 113-23.
21. Camarano AA. O idoso brasileiro no mercado de trabalho. Brasília: Instituto de Pesquisa Econômica Aplicada; 2001. (Texto para Discussão, 830).

22. Souza LJ, Chalita FEB, Reis AFF, Teixeira CL, Gicovate Neto C, Bastos A, et al. Prevalência de diabetes mellitus e fatores de risco em Campos dos Goytacazes, RJ. Arq Bras Endocrinol Metab 2003; 47:69-74.

23. Himes CL. Obesity, disease, and functional limitation in later life. Demography 2000; 37:73-82.

24. Zaitune MPA, Barros MBA, Cesar CLG, Carandina L, Goldbaum M. Fatores associados ao sedentarismo no lazer em idosos, Campinas, São Paulo, Brasil. Cad Saúde Pública 2007; 23:1329-38.

25. Tuomilehto J, Lindström J, Eriksson JG, Valle TT, Hämäläinen H, Ilanne-Parikka P, et al. Prevention of type 2 diabetes mellitus by changes in lifestyle among subjects with impaired glucose tolerance. N Engl J Med 2001; 344:1343-50.

26. Wing RR, Venditti E, Jakicic JM, Polley BA, Lang W. Lifestyle intervention in overweight individuals with a family history of diabetes. Diabetes Care 1998; 21:350-9.

27. Faria AN, Zanella MT. Tratamento de diabetes e hipertensão no paciente obeso. Arq Bras Endocrinol Metab 2002; 46:137-42.

28. Vlagopoulos PT, Tighiouart H, Weiner DE, Griffith J, Pettitti D, Salem DN, et al. Anemia as a risk factor for cardiovascular disease and all-cause mortality in diabetes: the impact of chronic kidney disease. J Am Soc Nephrol 2005; 16:3403-10.

29. Romão Junior JE. Doença renal crônica: definição, epidemiologia e classificação. J Bras Nefrol 2004; 26(3 Suppl 1):1-3.

30. Thomas M, MacIsaac R, Tsalamandris C, Power D, Jerums G. Unrecognized anemia in patients with diabetes: a cross-sectional survey. Diabetes Care 2003; 26:1164-9.

31. Lima MG, Barros MBA, Cesar CLG, Goldbaum M, Carandina, L, Ciconelli RM. Impact of chronic disease on quality of life among elderly in the state of São Paulo, Brazil: a population-based study. Rev Panam Salud Pública 2009; 25:314-21.

32. Korten AE, Jorm AF, Jiao Z, Letenneur L, Jacomb PA, Henderson AS, et al. Health, cognitive, and psychosocial factors as predictors of mortality in an elderly community sample. J Epidemiol Community Health 1999; 53:83-8.

33. Francisco PMSB, Donalisio MR, Barros MBA, Cesar CLG, Carandina L, Goldbaum M. Fatores associados à vacinação contra influenza em idosos. Rev Panam Salud Pública 2006; 19:359-64.

34. Centro de Vigilância Epidemiológica "Prof. Alexandre Vranjac”, Secretaria de Estado de Saúde de São Paulo. Campanha nacional de vacinação contra influenza 2007. São Paulo: Secretaria de Estado de Saúde de São Paulo; 2007.

Recebido em 21/Mai/2009

Versão final reapresentada em 01/Out/2009 Aprovado em 21/Out/2009 